\title{
Heating and Cooling Anomaly of a Rotating Body
}

O. Brůha, T. Brůha

This paper deals with an effect which appears when heating or cooling a rotating body. No external forces acting on the body are supposed. Due to thermal expansion, the moment of inertia of the body varies together with the temperature changes. In agreement with the principle of conservation of angular momentum [1], the angular momentum is constant. This results in angular velocity changes and subsequently in kinetic energy changes. Also the stress energy varies together with the changes in thermal dimension. To satisfy the principle of energy conservation we have to suppose that the changes in kinetic and stress energy are compensated by the changes in internal energy, which is correlated with temperature changes of the body. This means that the rules for the heating or cooling process of a rotating body are not the same as those for a body at rest. This idea, applied to a cylinder rotating around its geometric axis under specific parameters, has been mathematically treated. As a result, the difference between the final temperature of the rotating cylinder and the temperature of the cylinder at rest has been found.

Keywords: angular momentum, energy conservation, rotating body, thermal expansion.

\section{Introduction}

If a rotating body is heated, its internal energy increases and the concurrent temperature increase is followed by thermal expansion. This results in an increase in the moment of inertia of the body. When no external torque affects the body, then according to the principle of conservation of angular momentum, the angular momentum is constant [1]. Since the angular momentum of the body is proportional to the moment of inertia and to the angular velocity [1], the increase in the moment of inertia is followed by a reduction in angular velocity. The final effect is that there is a decrease in the kinetic energy of the rotating body, which is proportional to the moment of inertia and to the power in the angular velocity. (An example of a converse effect is the increase of the angular velocity of a "black hole" during its gravitational collapse).

According to the energy conservation law, the decay of kinetic energy must convert into another form of energy. Only thermal energy and deformation energy originating from centrifugal forces are in consideration. However, as proved by the following mathematical calculation, deformation energy also decreases with thermal expansion. This means that the deficit of kinetic energy and of deformation energy converts into an increase in the internal energy of the body. In sum, if a rotating body is heated, the increase in its internal energy is higher than if the body at rest. The "additional" increase in the internal energy of a rotating body appears to the detriment of its kinetic and deformation energy. In the following study the case of heating or cooling a rotating body is treated mathematically, and the trend of the quantities characterising the effect is demonstrated under concrete parameters.

\section{Assumptions}

It is supposed that the body, rotating with angular velocity $\omega_{0}$, (subscript "0" always means the initial value of a variable) is a cylinder with radius $r_{0}$, mass $m$ and height $h_{0}$, which is small in comparison with radius $r_{0}$. The axis of rotation and the geometric axis of the cylinder are identical. The density of the cylinder material is $\rho_{0}$, Young's modulus E, Poisson's number $\mu$, specific heat capacity c, and coefficient of thermal expansion $\alpha$ (Fig. 1). Quantities E, c, $\alpha$ are constant in the considered range of temperatures (approximately

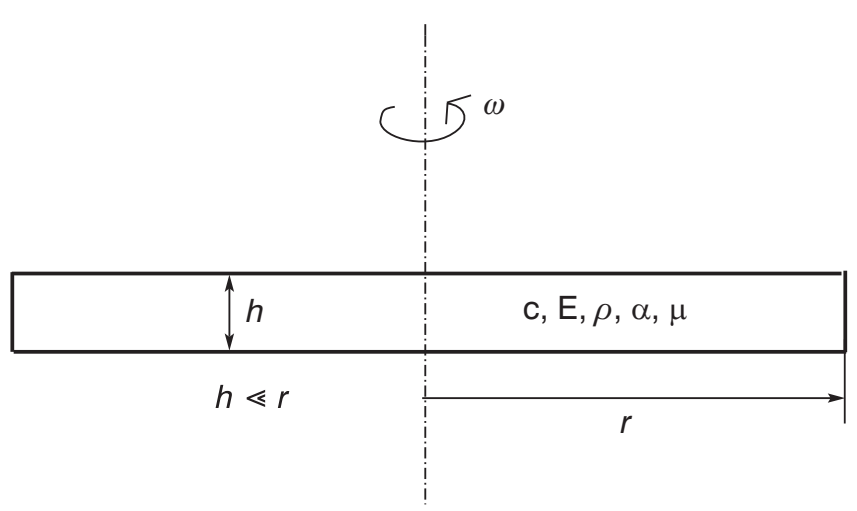

Fig. 1: A rotating cylinder and its parameters

300-1000 K) and the tension stress in the material does not exceed the proportional limit. No external forces affect the body and no uncontrolled heat transfer occurs between the cylinder and the environment (Fig. 2). For the parameters considered, the elastic deformation of the body originating from centrifugal forces is very small in comparison with the thermal expansion, and therefore its dependence on angular velocity is neglected.

\section{Mathematical treatment}

It is supposed that a quantity of heat $\Delta Q$ is transferred into or out of a rotating body (for instance by radiation, see Fig. 2). A mathematical description of the consecutive behaviour of the body follows. 


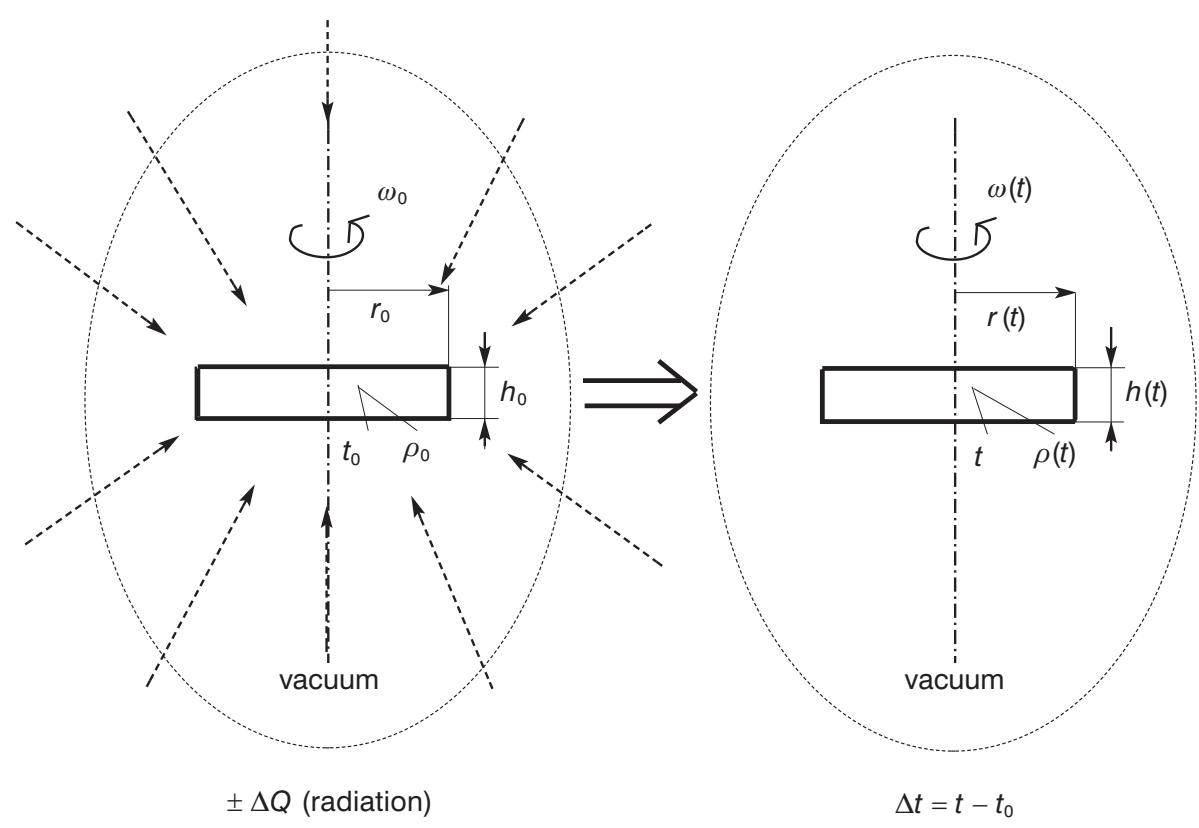

Fig. 2: An illustration of an isolated rotating body

\section{Basic relations}

Moment of inertia of the cylinder

$$
J=\frac{1}{2} m r^{2}
$$

Rotational kinetic energy of the cylinder

$$
E_{\mathrm{k}}=\frac{1}{2} J \omega^{2} .
$$

Angular momentum

$b=J \omega=$ const. (if the external torque is zero) [1], [2]

The linear coefficient of thermal expansion is defined generally as:

$$
\alpha=\frac{1}{x_{0}} \frac{\mathrm{d} x}{\mathrm{~d} t},
$$

where $x_{0}$ is initial length and $\mathrm{d} t$ is temperature change.

For better mathematical access another definition was used, namely:

$$
\alpha=\frac{1}{x} \frac{\mathrm{d} x}{\mathrm{~d} t},
$$

where $x$ is variable.

Relations (4) and (4a) result in practically the same values for the parameters used.

(For $\Delta t=700 \mathrm{~K}$ and $\alpha=16 \cdot 10^{-6} \mathrm{~K}^{-1}$, the difference in $\Delta \mathrm{x}$ calculated according to (4a) or according to (4) is only $0.0089 \Delta x$.)

Using relations (1) and (2) we can express the rotational kinetic energy of the cylinder (see Fig. 1.)

$$
E_{\mathrm{k}}=\frac{1}{4} \pi \rho h r^{4} \omega^{2} \text {. }
$$

For $r(t)$ and $h(t)$ as functions of temperature we obtain from definition (4):

$$
\begin{aligned}
& r(t)=r_{0} e^{\alpha \Delta t}, \\
& h(t)=h_{0} e^{\alpha \Delta t},
\end{aligned}
$$

where $\Delta t=t-t_{0}$.

Density $\rho(t)$ and angular velocity $\omega(t)$ as functions of temperature can be expressed in a similar way (see Appendix, items 1. and 2.)

$$
\begin{aligned}
& \rho(t)=\rho_{0} e^{-3 \alpha \Delta t}, \\
& \omega(t)=\omega_{0} e^{-2 \alpha \Delta t} .
\end{aligned}
$$

Substituting relations (6), (7), (8) and (9) in (5) we obtain an expression for rotational kinetic energy as a function of temperature

$E_{\mathrm{k}}(t)=\frac{1}{4} \pi r_{0}^{4} \omega_{0}^{2} h_{0} \rho_{0} e^{-2 \alpha \Delta t}=E_{\mathrm{k} 0} e^{-2 \alpha \Delta t}$.

The deformation energy should also be taken into consideration. The total deformation energy corresponding to the centrifugal forces in a rotating cylinder is (see Appendix, item 3.)

$$
\begin{aligned}
E_{\sigma} & =\frac{\pi \rho^{2} \omega^{4} r^{7} h}{448 \mathrm{E}}\left[\left(\frac{2}{3}-\frac{\mu}{2}\right)(3+\mu)^{2}\right. \\
& \left.+(3+\mu)(1+3 \mu)\left(\frac{\mu}{6}-\frac{1}{2}\right)+\frac{1}{6}(1+3 \mu)^{2}\right] .
\end{aligned}
$$

Substituting relations (6), (7), (8) and (9) in (11) we obtain for deformation energy as a function of temperature:

$$
\begin{aligned}
E_{\sigma}(t) & =\frac{\pi \rho_{0}^{2} \omega_{0}^{4} r_{0}^{7} h_{0} e^{-6 \alpha \Delta t}}{448 \mathrm{E}}\left[\left(\frac{2}{3}-\frac{\mu}{2}\right)(3+\mu)^{2}\right. \\
& \left.+(3+\mu)(1+3 \mu)\left(\frac{\mu}{6}-\frac{1}{2}\right)+\frac{1}{6}(1+3 \mu)^{2}\right] \\
& =E_{\sigma 0} e^{-6 \alpha \Delta t} .
\end{aligned}
$$

The total mechanical energy is

$$
E_{\sigma}(t)+E_{\mathrm{k}}(t)=E_{\sigma+\mathrm{k}}(t) .
$$

Using relations (10) and (12), the change in total mechanical energy as a function of temperature can be expressed as: 


$$
\begin{aligned}
\Delta E_{\sigma+\mathrm{k}}(t) & =E_{\sigma}(t)+E_{\mathrm{k}}(t)-E_{\sigma 0}-E_{\mathrm{k} 0} \\
& =E_{\mathrm{k} 0}\left(e^{-2 \alpha \Delta t}-1\right)+E_{\sigma 0}\left(e^{-6 \alpha \Delta t}-1\right) .
\end{aligned}
$$

Now we suppose that a quantity of heat $\Delta Q$ is added to (or taken away from) the rotating cylinder and we will try to express the corresponding change in its temperature $\Delta t$ according to equation (14). After the thermal field in the cylinder reaches the steady state, the angular velocity of the cylinder is again constant. There are no more reasons for changes to it. This means that the cylinder is in thermodynamic equilibrium both at the beginning of the process and also at the end [4]. Consequently, these two states must satisfy the principle of conservation of energy. Taking into account that not only mechanical energy, but also the change in internal energy $\Delta U$ has to be considered, the law of conservation of energy can be written as

$$
\Delta Q=\Delta U+\Delta E_{\sigma+\mathrm{k}},
$$

and

$$
\Delta U=\Delta Q-\Delta E_{\sigma+\mathrm{k}} .
$$

For a body at rest, it is generally supposed that:

$$
\Delta U_{0}=\Delta Q \text {. }
$$

This means that the change in internal energy, originating from the heat transferred $\Delta Q$, is not the same for a rotating body and for the same body at rest. There exists a difference

$$
\Delta^{\prime} U=\Delta U-\Delta U_{0}=\Delta Q-\Delta E_{\sigma+\mathrm{k}}-\Delta Q=-\Delta E_{\sigma+\mathrm{k}} \text {. }
$$

It is well known that a change in internal energy is correlated with a temperature change in a body. [1]. This means that a change in temperature corresponding to $\Delta \mathrm{Q}$ of a rotating body also differs from that of a body at rest.

Let us accept a simplified presumption, that the internal energy of a rigid body is entirely a function of the kinetic energy of chaotic motion of all particles and that an increase in it corresponds to an increase in the body temperature. Then we obtain a simplified expression for the difference $(\Delta * t)$ :

$$
\Delta * t=\Delta t-\Delta t_{\mathrm{s}}=\frac{\Delta^{\prime} U}{m \mathrm{c}} \quad[1],
$$

where $\Delta t$ is a change in temperature of the rotating body, and $\Delta t_{\mathrm{s}}$ is a change in temperature of the still body, $m$ is mass and $\mathrm{c}$ is specific heat capacity of the body.

Equation (19) combined with (14) and (18) yields:

$$
\Delta * t=\frac{E_{\mathrm{k} 0}\left(1-e^{-2 \alpha \Delta t}\right)+E_{\sigma 0}\left(1-e^{-6 \alpha \Delta t}\right)}{m \mathrm{c}} .
$$

According to (19)

$$
\Delta t=\Delta t_{\mathrm{s}}+\Delta * t
$$

and then $(20)$ reads:

$\Delta * t=\frac{E_{\mathrm{k} 0}\left(1-e^{-2 \alpha\left(\Delta^{*} t+\Delta t_{\mathrm{s}}\right)}\right)+E_{\sigma 0}\left(1-e^{-6 \alpha\left(\Delta^{*} t+\Delta t_{\mathrm{s}}\right)}\right)}{m \mathrm{c}}$,

where $\Delta t_{\mathrm{s}}$ is:

$$
\Delta t_{\mathrm{s}}=\frac{\Delta Q}{m \mathrm{c}}
$$

Expression (21) is a transcendent equation for unknown quantity $\Delta * t$ and given quantity $\Delta t_{\mathrm{s}}$. This equation was solved with the help of the MAPLE mathematical program, and the following real solutions for $\Delta * t$ as a function of $\Delta t_{\mathrm{s}}$ were found:

$$
\begin{aligned}
& \Delta * t=f\left(\Delta t_{\mathrm{s}}\right)= \\
= & -\frac{E_{\sigma 0} \cdot e^{6 \cdot \operatorname{RootOf}\left(Z m \mathrm{c}-\alpha E_{\sigma 0} e^{2} Z-\alpha E_{\mathrm{k} 0} e^{2} Z+\alpha E_{\sigma 0}+\alpha E_{\mathrm{k} 0}+\alpha \Delta t_{\mathrm{s}} m \mathrm{c}\right)}}{m \mathrm{c}} \\
& -\frac{E_{\mathrm{k} 0} \cdot e^{2 \cdot \operatorname{RootOf}\left(Z m \mathrm{c}-\alpha E_{\sigma 0} e^{6} Z-\alpha E_{\mathrm{k} 0} e^{6} Z+\alpha E_{\sigma 0}+\alpha E_{\mathrm{k} 0}+\alpha \Delta t_{\mathrm{s}} m \mathrm{c}\right)}}{m \mathrm{c}} \\
& -\frac{E_{\sigma 0}+E_{\mathrm{k} 0}}{m \mathrm{c}}
\end{aligned}
$$

(The symbol $Z$ in this equation represents an auxiliary variable for the solution of partial expressions for given $\Delta t_{\mathrm{s}}$.)

The solution (23) is illustrated (Fig. 3) for the following parameters of the rotating cylinder:

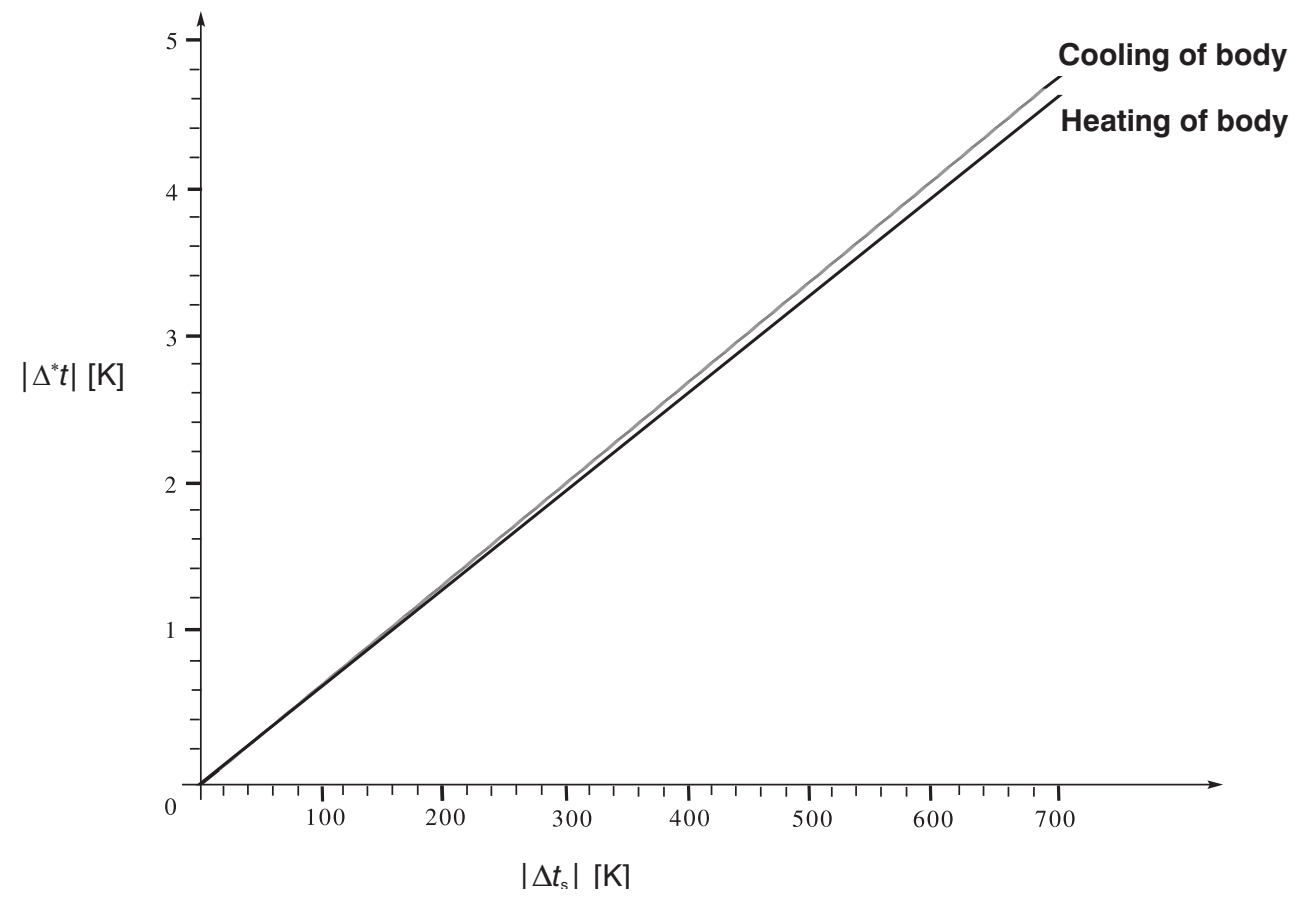

Fig. 3: Curves $\Delta^{*} t$ versus $\Delta t_{\mathrm{s}}$ as a solution of the equation (23) 
material - steel

$r_{0}=1 \mathrm{~m}$

$\omega_{0}=2 \pi 100 \mathrm{rad} \times \mathrm{s}^{-1}$

$h_{0}=0.1 \mathrm{~m}$

$\rho_{0}=7800 \mathrm{~kg} \times \mathrm{m}^{-3}$

$\mathrm{E}=2 \times 10^{11} \mathrm{~Pa}$

$a=16 \times 10^{-6} \mathrm{~K}^{-1}$

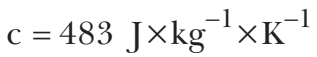

$\mu=0.29$

\section{Conclusions}

Fig. 3 shows the curves of $\Delta * t$ versus $\Delta t_{\mathrm{s}}$ (in absolute values) as a solution of equation (23), both for heating and for cooling the cylinder. For $\Delta t_{\mathrm{s}_{\max }}=700 \mathrm{~K}$, and for the parameters supposed, the value of $\Delta * t$ is:

$\Delta * t(700 \mathrm{~K})=4.62 \mathrm{~K}$ for heating and

$\Delta * t(700 \mathrm{~K})=4.72 \mathrm{~K}$ for cooling.

The results show that the effect is more readable for cooling than for the heating process.

This corresponds to the fact that for the heating process the temperature difference $\Delta * t$ has the finite asymptotic value:

$\Delta * t_{(\Delta t \rightarrow \infty)}=\frac{E_{\mathrm{k} 0}+E_{\sigma 0}}{m \mathrm{c}} \quad$ (follows from $(21)$ ).

(This relation describes a theoretical extreme case when the whole initial kinetic and deformation energy converts into inner energy and the cylinder "comes to a standstill").

In the case of cooling, the mathematical expression for $\Delta * t$ has no limit.

In the study it is supposed that the coefficient of thermal expansion of the cylinder material is positive. If the coefficient of thermal expansion is negative, the heating of a rotating cylinder would result in an increase in kinetic energy on account of the heat transferred. As materials with a negative coefficient of thermal expansion are very rare (for instance quenched irreversible Fe-Ni invar alloys), this case is not considered in detail in the study.

\section{Appendix}

\section{Relation (8):}

From its definition, the density is given by:

$$
\rho=\frac{m}{V}
$$

and according to Fig. 1:

$$
\rho=\frac{m}{\pi r^{2} h},
$$

or:

$$
\rho_{0}=\frac{m}{\pi r_{0}^{2} h_{0}}, \quad \rho(t)=\frac{m}{\pi r^{2}(t) h(t)},
$$

and from relation $(6,7)$ follows:

$\rho(t)=\frac{m}{\pi r_{0}^{2} e^{2 \alpha \Delta t} h_{0} e^{\alpha \Delta t}}=\frac{m}{\pi r_{0}^{2} h_{0} e^{3 \alpha \Delta t}}=\rho_{0} e^{-3 \alpha \Delta t}$.

\section{Relation (9):}

According to relation (3):

$$
J \omega=\text { const. }
$$

or:

$$
\begin{aligned}
& J_{0} \omega_{0}=J(t) \omega(t), \\
& \Rightarrow \omega(t)=\frac{J_{0}}{J(t)} \omega_{0}
\end{aligned}
$$

and from relation $(1,6)$ follows:

$\omega(t)=\frac{\frac{1}{2} m r_{0}^{2}}{\frac{1}{2} m r_{0}^{2} e^{2 \alpha \Delta t}} \omega_{0}=\omega_{0} e^{-2 \alpha \Delta t}$.

\section{Relation (11):}

Note: since the height of the cylinder is small in comparison with its radius, the axial stress is neglected.

The deformation energy $\mathrm{E}_{\sigma}$ per volume unit corresponding to the tension stress $\sigma$ is:

$$
\frac{E_{\sigma}}{V}=\frac{\sigma^{2}}{2 \mathrm{E}}
$$

and

$$
E_{\sigma}=\frac{\sigma^{2} V}{2 \mathrm{E}}
$$

and in elementary form:

$$
\mathrm{d} E_{\sigma}=\frac{\sigma^{2} \mathrm{~d} V}{2 \mathrm{E}} .
$$

In the case that the height of the rotating cylinder is small in comparison with its radius, radial and tangential stress $\sigma_{\mathrm{r}}$ and $\sigma_{\mathrm{t}}$, originating from centrifugal force, can be expressed as (see Fig. 4)

$$
\sigma_{\mathrm{r}}\left(r_{x}\right)=\frac{3+\mu}{8} \rho \omega^{2}\left(r^{2}-r_{x}^{2}\right)
$$

and

$\sigma_{\mathrm{t}}\left(r_{x}\right)=\frac{\rho \omega^{2}}{8}\left(r^{2}(3+\mu)-r_{x}^{2}(1+3 \mu)\right)$,

where $r_{x}$ is the radius of stress action.

The elementary deformation energy corresponding to the radial and tangential stress is

$\mathrm{d} E_{\sigma}\left(r_{x}\right)=\frac{\mathrm{d} V}{2 \mathrm{E}}\left(\sigma_{\mathrm{r}}\left(r_{x}\right)^{2}+\sigma_{\mathrm{t}}\left(r_{x}\right)^{2}-2 \mu \sigma_{\mathrm{r}}\left(r_{x}\right) \sigma_{\mathrm{t}}\left(r_{x}\right)\right)$.

As shown in Fig. 4.:

$$
\mathrm{d} V=\mathrm{d} l S=r_{x} \mathrm{~d} \varphi h \mathrm{~d} r .
$$

Substituting relation (A15) in (A14) we obtain for elementary deformation energy:

$$
\begin{aligned}
\mathrm{d} E_{\sigma}\left(r_{x}\right)=\frac{h}{2 \mathrm{E}} & r_{x}\left(\sigma_{\mathrm{r}}\left(r_{x}\right)^{2}+\sigma_{\mathrm{t}}\left(r_{x}\right)^{2}-\right. \\
& \left.-2 \mu \sigma_{\mathrm{r}}\left(r_{x}\right) \sigma_{\mathrm{t}}\left(r_{x}\right)\right) \mathrm{d} r \mathrm{~d} \varphi .
\end{aligned}
$$

Substituting relations (A12), (A13) in (A16) and integrating we obtain the integral deformation energy accumulated in the rotating cylinder: 


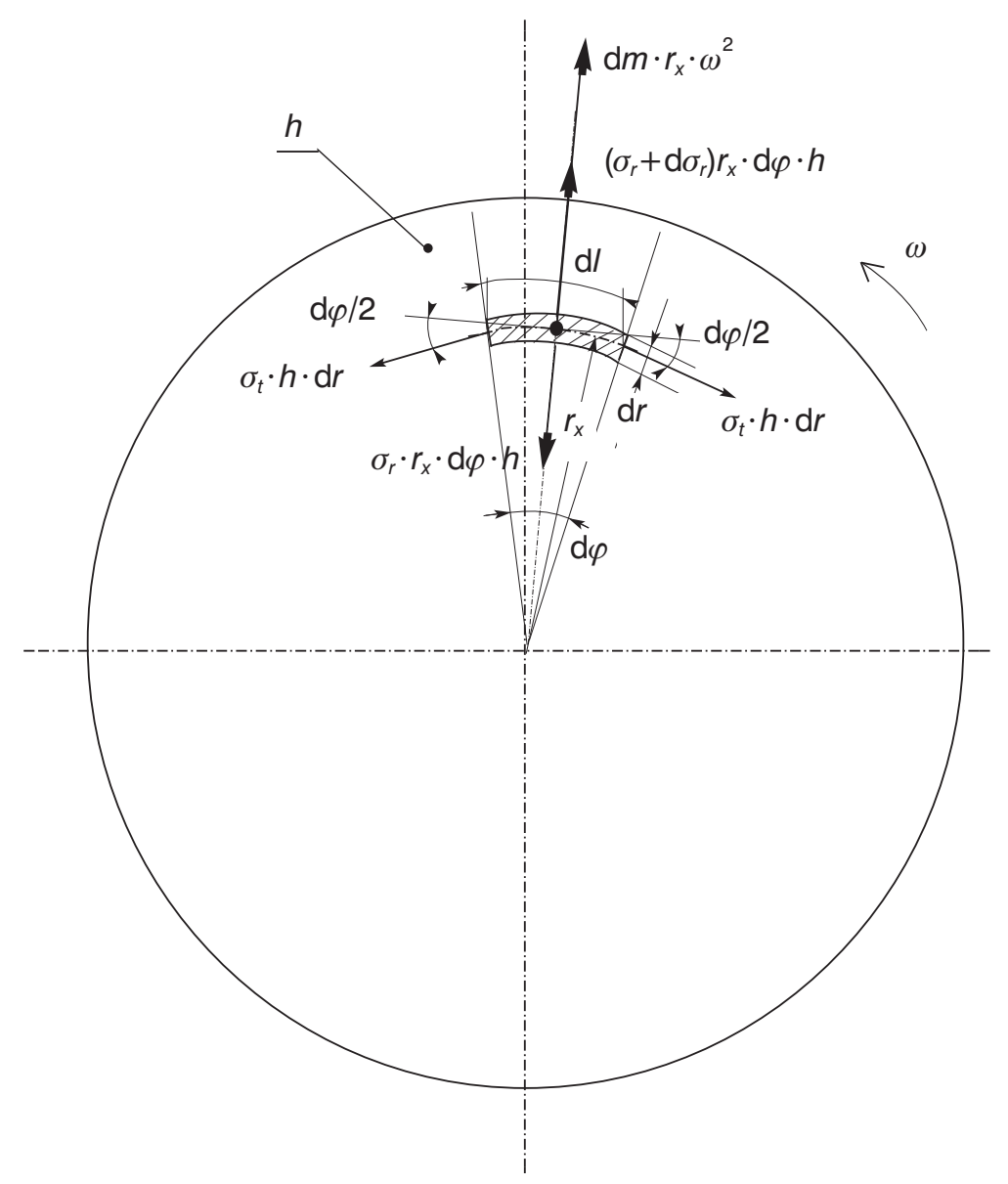

Fig. 4: Centrifugal and stress forces in a rotating cylinder

$$
\begin{aligned}
E_{\sigma}= & \int_{0}^{r} \int_{0}^{2 \pi} \mathrm{d} E_{\sigma}\left(r_{x}\right) \mathrm{d} r \mathrm{~d} \varphi \\
= & \frac{\pi \rho^{2} \omega^{4} r^{7} h}{448 \mathrm{E}}\left(\left(\frac{2}{3}-\frac{\mu}{2}\right)(3+\mu)^{2}\right. \\
& \left.+(3+\mu)(1+3 \mu)\left(\frac{\mu}{6}-\frac{1}{2}\right)+\frac{1}{6}(1+3 \mu)^{2}\right) .
\end{aligned}
$$

\section{References}

[1] Tipler, P. A.: "Physics." New York, Worth Publisher Inc., 1982, p. 80, 295-297, 495-496, 498-500.

[2] Horák, Z., Krupka, F., Šindelář, V.: „Technická fyzika.“ Praha, SNTL, 1960, p. 187-188, 310, 1411.

[3] Michalec, J. et al.: „Pružnost a pevnost II.“ Praha, Vydavatelství ČVUT, 2001, p. 78-82.

[4] Landau, L. D., Lifschitz, E. M.: "Lehrbuch der Theoretischen Physik V: Statistische Physik." Berlin, Akademie-Verlag, 1966, Kap. 2, § 10, p. 38-40.
Doc. Ing. Oldřich Brůha, CSc. phone: +420602458314 fax: +420251561115 e-mail: oldrich.bruha@volny.cz

Department of Physics

Czech Technical University in Prague Faculty of Mechanical Engineering Technická 4

16607 Praha 6, Czech Republic

Ing. Tomáš Brůha phone: +420777895762

e-mail: enex@volny.cz

ENEX s.r.o.

Na Míčánce 13

16000 Praha 6, Czech Republic 